\title{
Solar Flare Particle Propagation: Comparison of a New Analytic Solution with Spacecraft Measurements
}

\author{
J. E. LUPTON ${ }^{1}$ AND E. C. Stone
}

\author{
California Institute of Technology, Pasadena, California 91109
}

\begin{abstract}
A new radial solution has been obtained to the Fokker-Planck equation for solar flare particle propagation that includes the effects of convection, energy change, Ind anisotropic diffusion with $\kappa_{r}=$ constant. It is assumed that the particles are injected impulsively at a single point and that there is a free escape boundary. In addition, the azimuthal solution derived by Burlaga, which was based on $\kappa_{\theta} \propto r^{2}$, has been modified to include some of the effects of solar rotation. With an outer boundary at $\sim 2.7 \mathrm{AU}$, a solar wind velocity of $\sim 400$ $\mathrm{km} / \mathrm{sec}$, and $\kappa_{\tau} \approx 2$ to $8 \times 10^{30} \mathrm{~cm}^{2} / \mathrm{sec}$, the complete solution gives reasonable fits to the time profiles of 1- to 10-Mev protons from 'classical' flare-associated events ôbserved with the Caltech solar and galactic cosmic ray experiment aboard Ogo 6. It is not necessary to invoke a scatter free region near the sun in order to reproduce the fast rise times observed for west limb events, indicating that $k_{r}=$ constant is a better description of conditions inside $1 \mathrm{AU}$ than is $\kappa_{r} \propto r$. The radial solution also provides insight into the possible dependence of the observed decay times on various parameters and can be used as the basis for an illustrative calculation of the evolution of the vector anisotropy.
\end{abstract}

Solar flare particle transport has been studied both theoretically and experimentally for a number of years. Parker [1965], who correctly described the general features of interplanetary space (a spiral magnetic field imbedded in an outward-flowing solar wind), also formulated the propagation of energetic particles through this medium in terms of the Fokker-Planck equation:

$$
\begin{aligned}
\frac{\partial U}{\partial t}=\nabla \cdot(\kappa & \cdot \nabla U)-\nabla \cdot(U \mathrm{~V}) \\
& +\frac{1}{3} \nabla \cdot \mathbf{v}\left\{\frac{\partial}{\partial T}(\alpha(T) T U)\right\}
\end{aligned}
$$

where $U$ is the differential particle density per unit energy interval, $r$ is the radial distance from the sun, $\mathbf{V}$ is the solar wind velocity (assumed to be independent of spatial parameters), $\alpha(T)=$ $\left(T+2 M_{0} c^{2}\right) /\left(T+M_{0} c^{2}\right)$, and $\boldsymbol{x}$ is the diffusion tensor describing the random walk of the particles in the interplanetary plasma. This equation, which describes the particle density in a stationary frame of reference, includes the effects of

1 Now at the Scripps Institution of Oceanography, La Jolla, California.

Copyright (c) 1973 by the American Geophysical Union. diffusion, outward convection by the solar wind, and adiabatic cooling due to the solar wind expansion. Further derivations are given by Jokipii and Parker [1967] and Gleeson and Axford [1967].

Many solutions to the Fokker-Planck equation have been developed in an effort to understand the particle fluxes observed after solar flare injection. Burlaga [1967] has produced a solution to the diffusion equation assuming anisotropic diffusion within a region surrounded by a free escape boundary. His solution describes observations of $\gtrsim 100$-Mev protons adequately, but neglects the effects of convection and adiabatic deceleration, which are important at lower energies [Forman, 1970, 1971a]. More recently, Fisk and Axford [1968], Forman [1971a], and $N g$ and Gleeson [1971a, b] have discussed analytic solutions that include impulsive injection, diffusion, convection, and energy change. Forman's [1971a] solution, which employs a bounded diffusing region with $\kappa_{r} \propto r$ and $\kappa_{\theta} \propto r^{2}$, adequately describes the decay rate of low-energy solar flare events, but predicts a time to maximum that is longer than the $\sim 15$ hours frequently observed for west limb events.

Such discrepancies between theory and observation are the result of the approximations and assumptions necessarily invoked to obtain a 
solution to the equation. Since many of these assumptions cannot be directly tested, such discrepancies are often the only indication of the validity of the assumptions. In the discussion that follows, a slightly modified set of assumptions results in a solution that is in better agreement with the observations. Detailed comparisons between this solution and actual observations will be made that yield information about (a) the diffusion tensor; (b) the azimuthal propagation of particles; $(c)$ the existence of an outer boundary to the diffusion region; $(d)$ scatter free propagation near the sun; $(e)$ the vector particle anisotropy; and $(f)$ the energy change effect. The results not only provide insight into the assumptions, but also indicate how the assumptions might be modified to obtain a better solution.

\section{Solution FoR Constant $\kappa_{r}$}

The new solution, which was the subject of a preliminary report [Lupton and Stone, 1971], employs the following boundary conditions and simplifying assumptions:

(a) The differential particle density $U=$ $\partial n / \partial T$ depends only on spatial position $(r, \theta, \phi)$, time $t$, and particle kinetic energy $T$.

(b) All parameters except for the particle density $U$ are assumed to be independent of energy $T$.

(c) The solar wind velocity $\mathbf{V}$ is radial and independent of $(r, \theta, \phi, t)$.

(d) No attempt is made to describe transient phenomena such as solar wind fluctuations, shock waves, and hydromagnetic waves.

(e) The particles are impulsively injected at $r=r$, at time $t=0$ with an energy spectrum defined by $U_{0}(T)$. Specifically,

$$
\begin{aligned}
U(r, \theta, \phi, t & =0, T) \\
& =\frac{\delta\left(r-r_{s}\right)}{r^{2}} f(\theta, \phi) U_{0}(T)
\end{aligned}
$$

(f) The differential density $U$ remains finite as $r \rightarrow 0$.

(g) A perfectly absorbing boundary exists at $r=L$ such that $U(L, \theta, \phi, t, T)=0$.

(h) The diffusion tensor is taken to be diagonal in a reference frame aligned with the radial direction. In particular, $\boldsymbol{x}$ will be defined by $\kappa_{\theta}=\kappa_{\phi}=\kappa_{1} r^{2}$ and $\kappa_{r}=\kappa=$ constant, both independent of energy $T$.
The most critical assumptions are that $\boldsymbol{x}$ is independent of energy $T$ and is aligned with the principal axis in the radial direction. The energy dependence of $\kappa$ will be treated by dividing the energy domain into intervals within which $\kappa$ is independent of $T$. This approach will be justified later.

Some comment should be made about the assumed diagonal form for the diffusion tensor in the $(r, \theta, \phi)$ reference frame. This would be the correct form if the magnetic field were radial. Then $\kappa_{r}=\kappa_{\|}, \kappa_{\theta}=\kappa_{\phi}=\kappa_{\perp}$, and the variable $r$ would represent a path length measured along a field line. Note that, contrary to actual conditions, the convective effects are in this case parallel to the field lines. However, Englade [1971a], using a computational model for flare particle propagation, has found that approximating the spiral field with a radial configuration has only a small effect on the flux observed at $1 \mathrm{AU}$.

The boundary condition at the sun would seem to be more appropriately described by either a perfectly absorbing or perfectly reflecting boundary at $r=r$, (instead of at the origin). However, $\mathrm{Ng}$ and Gleeson [1971a] and Englade [1971b] have shown that the type of solar boundary condition assumed has little effect on the time profile observed at $1 \mathrm{AU}$.

The assumption of an outer absorbing boundary may not be necessary, and several authors have developed solutions using an infinite diffusion region. In particular, $\mathrm{Ng}$ and Gleeson [1971a] find that an approximately exponential decay can be produced assuming $\kappa_{\|}=\kappa_{0}(1+$ $\left.r^{a}\right)$. This $\kappa_{\| 1}$ function provides a smooth transition to a region of free escape, where $\kappa \rightarrow \infty$ and $U \rightarrow 0$. An abrupt boundary may be a reasonable approximation to use until more is learned about the $r$ dependence of $\kappa_{r}$. The $r$ dependence is a very important assumption, as will be demonstrated later in the discussion.

Applying the above simplifications and assuming $U(r, \theta, \phi, t, T)=R(r, t) Q(\theta, \phi, t) T^{-\gamma}$, the Fokker-Planck equation can be separated into the following equations:

$$
\begin{aligned}
& \frac{\kappa_{1}}{\sin \theta} \frac{\partial}{\partial \theta} \sin \theta \frac{\partial Q}{\partial \theta}+\frac{\kappa_{1}}{\sin ^{2} \theta} \frac{\partial^{2} Q}{\partial \phi^{2}}=\frac{\partial Q}{\partial t} \\
& \frac{1}{r^{2}} \frac{\partial}{\partial r}\left[r^{2} \kappa_{r}(r) \frac{\partial R}{\partial r}\right]-V \frac{\partial R}{\partial r}-\frac{2 C V}{r} R=\frac{\partial R}{\partial t}
\end{aligned}
$$


where the constant $C$ is the Compton-Getting factor

$$
C=1-\frac{1}{3 U} \frac{\partial}{\partial T}[\alpha(T) T U]
$$

which, for a power law proton spectrum at energies below $\sim 100 \mathrm{Mev}$, can be written

$$
C=(2 \gamma+1) / 3
$$

Note that it is not necessary to specify the form of $\kappa_{r}(r)$ to carry out the separation, but it is necessary to assume that $\kappa_{\theta}=\kappa_{1} r^{2}$ and that $\boldsymbol{k}$ is independent of energy $T$. If $U(r, \theta, \phi, t, T)$ is separable, the radial part of the solution $R(r, t)$ is independent of any additional assumptions about the azimuthal propagation.

For completeness we note that the solution can be generalized to describe any energy change process that varies as $1 / r$ by introducing a time constant $\tau_{B}(r)=\tau_{0} r$ which characterizes the strength of the energy change process. For pure adiabatic deceleration, $\tau_{0}=3 / 4 \mathrm{~V}$ and $C=(2 \gamma+1) / 3$. If some other energy change process is operative, then $\tau_{0}$ becomes a free parameter and $C=1-(\gamma-1) / 2 V_{\tau_{0}}$.

The solution for the azimuthal dependence (equation 3) is identical to that used by Burlaga [1967] and Forman [1971a]:

$$
\begin{aligned}
Q(\theta, \phi, t)=\sum_{l=0}^{\infty} & C_{l} P_{l}(\cos \delta) \\
& \cdot \exp \left[-l(l+1) \kappa_{1} t\right]
\end{aligned}
$$

where

$$
\begin{aligned}
\cos \delta= & \cos \theta \cos \theta_{0} \\
& +\sin \theta \sin \theta_{0} \cos \left(\phi-\phi_{0}\right)
\end{aligned}
$$

and $\left(\theta_{0}, \phi_{0}\right)$ is the center of the injection profile $f(\theta, \phi)$ at the sun. For the $\delta$ function injection, which will be used in this discussion, $C_{3}=$ $2 l+1$. Presumably the response to any symmetric $f(\theta, \phi)$ can be generated using the proper coefficients $C_{t}$. Since the sun is rotating, the center of the injection profile is moving with respect to the earth at the rate $\Omega t$, and the earth is connected to the sun by a magnetic field line displaced by an angle $\Omega r / V$ from the central meridian. These effects can be included in part by using

$\cos \delta=\cos \lambda_{0} \cos \left(\Phi_{0}-\Omega r / V+\Omega t\right)$ where $\lambda_{0}$ and $\Phi_{0}$ are the solar latitude and longitude of the optical flare, and $\Omega$ is the solar angular velocity of rotation. Note that Englade [1971a] has shown that the effects of solar rotation are more complicated than those discussed here.

The solution for the radial dependence (equation 4) requires an assumption for the form of $\kappa_{r}(r)$. If $\kappa_{r}(r)=\kappa$, equation 4 becomes

$$
\frac{\partial^{2} R}{\partial r^{2}}+\left(\frac{2}{r}-\frac{V}{\kappa}\right) \frac{\partial R}{\partial r}-\frac{2 C V}{\kappa \tau} R=\frac{1}{\kappa} \frac{\partial R}{\partial t}
$$

A solution to equation 10 has been found using the boundary conditions and assumptions listed above. The details of the derivation are given in Appendix A. This solution can be written as the following eigenvalue expansion:

$$
\begin{gathered}
R(r, t)=A \frac{\exp \left[V\left(r-r_{s}\right) / 2 \kappa\right]}{r_{s}} \\
\cdot \sum_{n=1}^{\infty} \frac{1}{N_{n}} F_{0}\left(\frac{\beta}{2\left(\alpha_{n}\right)^{1 / 2}},\left(\alpha_{n}\right)^{1 / 2} r_{s}\right) \\
\cdot F_{0}\left(\frac{\beta}{2\left(\alpha_{n}\right)^{1 / 2}},\left(\alpha_{n}\right)^{1 / 2} r\right) \exp \left(-\frac{t}{\tau_{n}}\right)
\end{gathered}
$$

where $F_{0}(\eta, \rho)$ is the regular Coulomb wave function [Abramowitz and Stegun, 1964]. The $\alpha_{n}$ are the eigenvalues defined by the outer boundary condition

$$
F_{0}\left(\beta / 2\left(\alpha_{n}\right)^{1 / 2},\left(\alpha_{n}\right)^{1 / 2} L\right)=0
$$

The other parameters are defined as follows:

$$
\begin{aligned}
\beta & =V(2 C-1) / \kappa \\
C & =1+(\gamma-1) / 2 V \tau_{0} \\
\tau_{n} & =4 \kappa /\left(4 \kappa^{2} \alpha_{n}+V^{2}\right) \\
N_{n} & =\int_{0}^{L}\left[F_{0}\left(\beta / 2\left(\alpha_{n}\right)^{1 / 2},\left(\alpha_{n}\right)^{1 / 2} x\right)\right]^{2} d x
\end{aligned}
$$

and $A$ is arbitrary normalization.

This expansion converges rapidly for $t \gtrsim 5$ hours for typical values of the other parameters. In the limit as $V / \kappa_{r} \rightarrow 0$ the solution reduces smoothly to the result obtained by Burlaga [1967].

Because equation 10 is linear, solutions can be superimposed, and one is not restricted to a pure power law energy dependence. Thus a more complicated initial spectrum $U_{0}(T)$, which can be written as a superposition of power laws 


$$
U_{0}(T)=\sum_{i} A_{i} T^{-\gamma_{i}}
$$

can be treated by writing the radial solution in a more general form:

$$
\begin{aligned}
& U(r, \theta, \phi, t, T) \\
& \quad=Q(\theta, \phi, t) \sum_{i} A_{i} T^{-\gamma} R_{i}(r, t)
\end{aligned}
$$

which consists of a sum over separate radial functions $R_{i}(r, t)$, each corresponding to a different power law index $\gamma_{i}$. The azimuthal dependence $Q(\theta, \phi, t)$ is not involved in this summation because the diffusion tensor $x$ is assumed in this model to be independent of energy $T$.

\section{Befavior of the Radial solution}

Because many features of the time profile of a solar flare event are due to the 'radial' transport of particles, a discussion of $R(r, t)$ may provide insight into actual particle propagation. Figure 1 shows the distribution of particles $R(r, t)$ as a function of radial distance $r$ at various times using typical values of the parameters. The peak in the particle distribution,

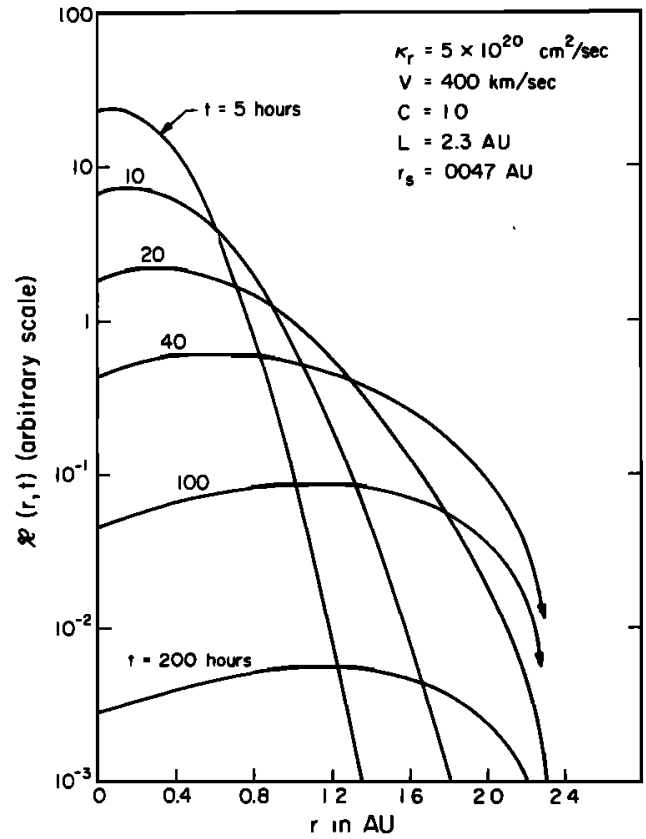

Fig. 1. The radial solution $R(r, t)$ defined by equation 11 is plotted versus radial distance $r$ for various times after particle injection at typical values of the parameters. which is initially near the sun due to the $\delta$ function injection, moves outward until the effect of the absorbing boundary is felt. Eventually the distribution assumes a stable shape. The profile $R(r, t)$ versus $t$ observed at any position $r$ displays a rapid rise to maximum intensity followed by a decay phase. The time to maximum, which depends on observation distance $r$, is $\sim 15$ hours at $1 \mathrm{AU}$. At late times ( $t \gtrsim 100$ hours), the first term of equation 11 dominates, and the intensity decays exponentially at a rate characterized by тово $=\tau_{1}$, which depends only on the boundary location $L$, solar wind speed $V$, diffusion coefficient $\kappa_{r}$, and the energy change parameter $C$.

Figure 2 demonstrates the dependence of $\tau_{\mathrm{DmC}}$ on $\kappa_{r}, L, V$, and $C$. Note that there is a range of values of $\kappa_{\text {r }}$ over which the decay rate is only weakly dependent on $\kappa_{r}$. An increase in $V$ hastens the decay because these convection and energy change effects are strengthened. As the ratio $V / \kappa_{r} \rightarrow 0$, the decay time constant approaches Burlaga's $\left(\kappa_{r}\right)^{-1}$ dependence as expected.

The decay in the particle intensity is produced by the outward transport of particles to the boundary and by the energy loss process. Increasing the boundary distance $L$ thus produces a marked decrease in the decay rate, because the processes of convection and diffusion deliver particles to the boundary less rapidly, and the energy change rate is slower at greater distances.

Figure 2(c), which shows the effects of the spectral index $\gamma$ on the decay rate of intensity, demonstrates a strong dependence on $\gamma$. This becomes clear when the radial transport equation is written as

$$
\begin{aligned}
\frac{1}{\tau_{\mathrm{DEC}}}=-\frac{1}{R} \frac{\partial R}{\partial t}= & -\frac{1}{R r^{2}} \frac{\partial}{\partial r}\left[r^{2}{ }_{\kappa_{r}}(r) \frac{\partial R}{\partial r}\right] \\
& +\frac{V}{R} \frac{\partial R}{\partial r}+\frac{2 C V}{r}
\end{aligned}
$$

The last term yields $\tau_{\mathbb{D C}}=r /(2 C V)$, which at $1 \mathrm{AU}$ is $\sim 17$ hours, of the same order as the total decay constant $\tau_{\text {nmo. }}$.

The decay rates shown in Figure 2 occur late in the event when the relative distribution of particles $f(r)$ is given by

$$
\begin{aligned}
f(r)=r^{-1} F_{0}\left(\beta / 2\left(\alpha_{1}\right)^{1 / 2},\right. & \left.\left(\alpha_{1}\right)^{1 / 2} r\right) \\
& \cdot \exp (V r / 2 \kappa)
\end{aligned}
$$



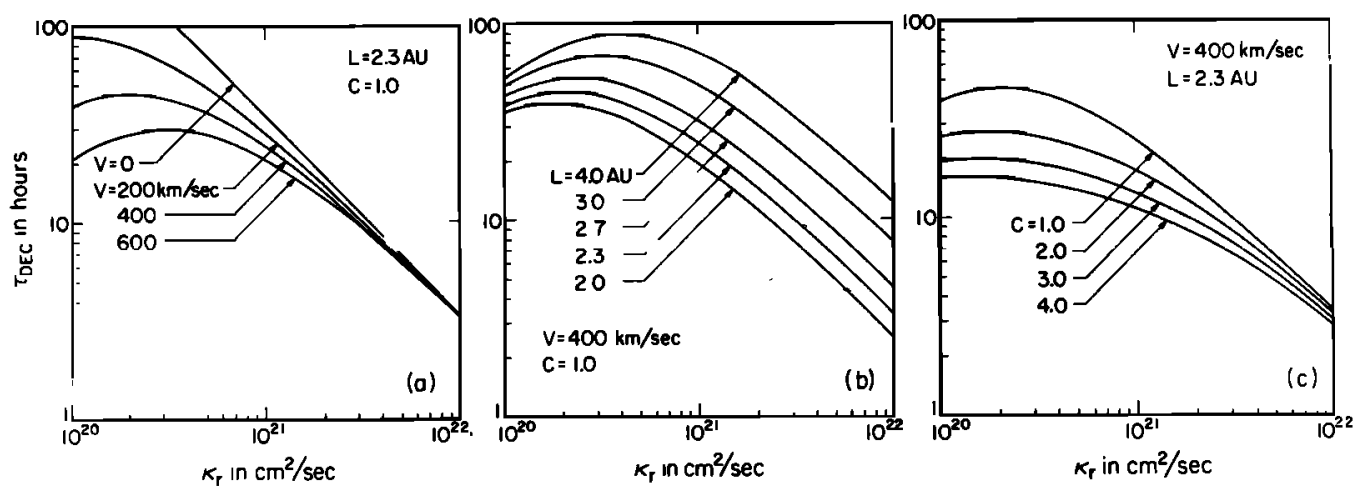

Fig. 2. The exponential decay time constant $\tau$ Dmo predicted by the radial solution is plotted versus $k_{\mathrm{r}}$ for $(a)$ various values of the solar wind velocity $V,(b)$ various values of the boundary position $L$, and $(c)$ different values of the energy change parameter $C$.

which depends only on $L, C$, and the ratio $V / k_{r}$. This function is shown in Figure 3 for various typical values of this ratio. Again as $V / \kappa_{r} \rightarrow 0$, $f(r)$ reduces to Burlaga's sin $(\pi r / L) / r$, which is peaked at the origin. As $V / \kappa_{r}$ is increased, the peak in the distribution becomes more pronounced and moves outward due to the increased convective effects. The dependence of the position of this peak on $V / \kappa$ has been discussed by Forman [1971b] for a model with $\kappa=\kappa_{0} r$.

If we know the distribution of particles at all times, we can calculate the particle current $S$ as a

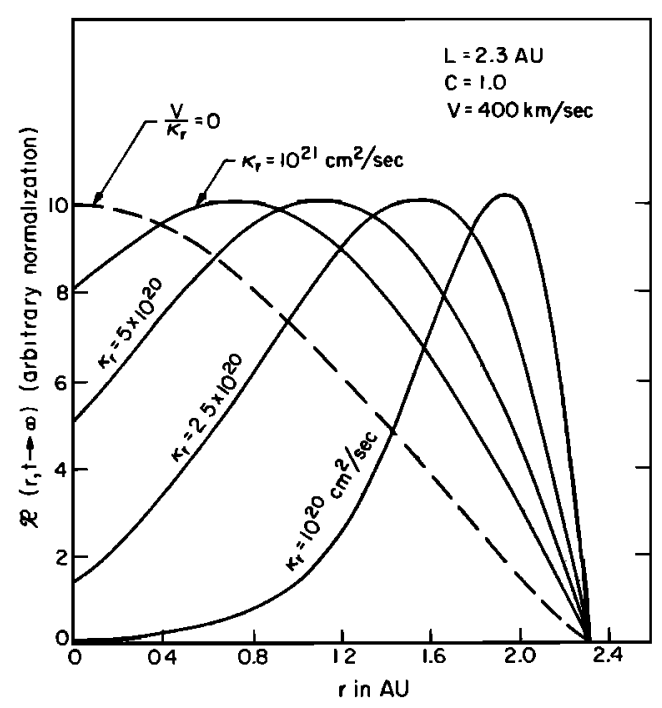

Fig. 3. The radial distribution of particles at late times is plotted versus $r$ for various values of the ratio $V / k_{r}$. function of time, where

$$
\mathbf{s}=C \mathbf{V} U-\boldsymbol{k} \cdot \boldsymbol{\nabla} U
$$

It is illustrative to consider the radial part of the streaming $S$, which can be written as a scalar composed of convective and diffusive terms as calculated from the radial part of the solution;

$$
S=S_{V}+S_{\kappa}=C V R-\kappa_{r} \frac{\partial R}{\partial r}
$$

In this discussion the streaming due to azimuthal gradients is neglected. Figure 4, which shows the decay phase values of $S, S_{\nabla}$, and $S_{\kappa}$ as a function of $r$, makes it clear which transport process is most important at any given radial distance. At the outer boundary, the net particle flux is outward and is due entirely to diffusion. Because the typical particle distribution at late times is peaked at $r \gtrsim 1 \mathrm{AU}$ (see Figure 3), the gradient over much of the distance is positive and the diffusive current $S_{\kappa}$ in this region is directed inward. This inward diffusion, which impedes the process of particle escape, exactly balances the outward convection at the origin to produce $S(r=0)=0$.

The decay time constant $\tau_{\mathrm{DEO}}=-U /(\partial U / \partial t)$ is a measure of the total stored particle population relative to the rate at which particles reach the boundary and escape. Figure 2 shows that a broad maximum in $\tau_{D \mathbb{O}}$ versus $\dot{\kappa}_{r}$ occurs typically for $\kappa_{r} \approx 2 \times 10^{20} \mathrm{~cm}^{2} / \mathrm{sec}$. This maximum also oecurs in the solution using $\kappa=\kappa_{0} r[$ For man, 1971b]. 


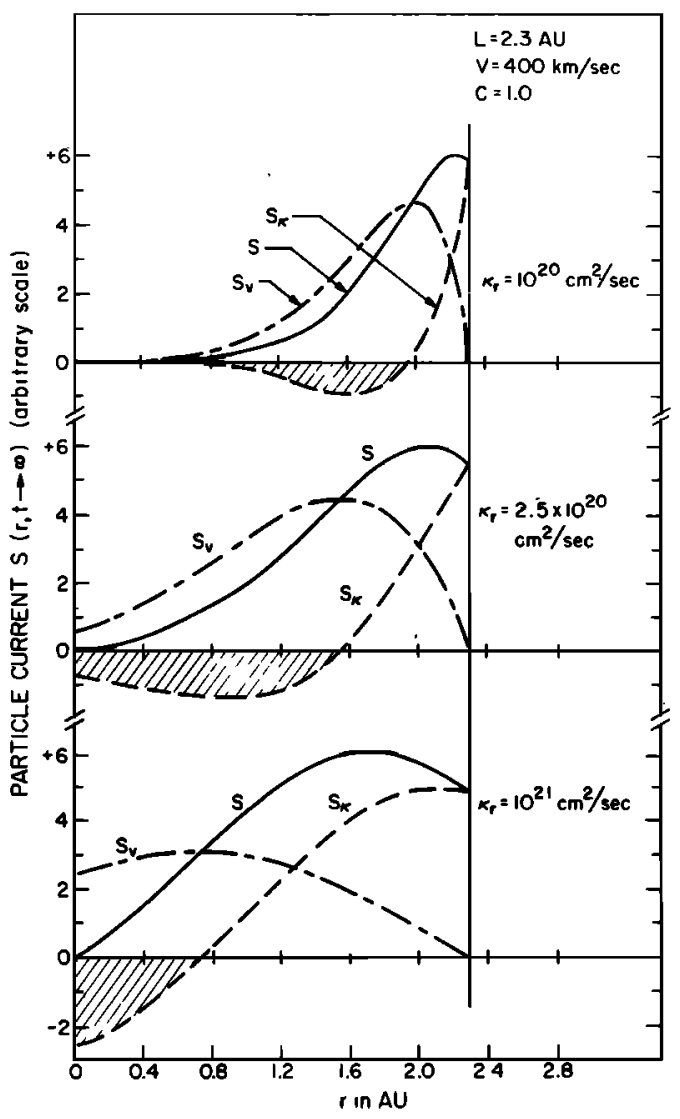

Fig. 4. The particle current $S$ at late times and the diffusive and convective components $S_{\kappa}$ and $S_{V}$ are plotted versus $r$ for three values of $x_{r}$. The shaded areas emphasize the region where the diffusive current $S_{\kappa}$ is inward.

\section{Fits to Actual Data Assuming Adiabatic Deceleration}

Additional insight into the approximations and parameters involved in the new solution results from comparison with three 'classical' prompt flare events that have been analyzed using the Caltech solar and galactic cosmic ray experiment [Althouse et al., 1967] aboard NASA's Ogo 6 spacecraft. Table 1 summarizes the pertinent data for each of these events.

Profiles of proton intensity versus time for various incident energy bins were fitted separately by eye with the new solution using only $\kappa_{r}, \kappa_{\theta}(1 \mathrm{AU})$, and $A$ as free parameters. The time and location $\left(\lambda_{0}, \Phi_{0}\right)$ of particle injection were derived from the parent flare identification for each event, while the average solar wind velocity $V$ was assigned from the Solar Geophysical Data (1969-1970). The radial position of injection was taken as the surface of the sun $\left(r_{a}=0.0047 \mathrm{AU}\right)$, and an observer position of $r=1.15$ AU was used, corresponding to the path length along the average spiral field. It was assumed that adiabatic deceleration was the only energy change process operative. Observations of the intensity within individual energy bins were fitted independently, and values of $C$ were assigned to each energy interval by finding the average $\gamma(T)=d(\ln U) / d(\ln T)$ over the 3-day observation period. Since the resulting values of $\kappa_{r}$ and the observed spectral index $\gamma$ have only a weak energy dependence, the initial assumption that $\kappa$ is independent of energy is reasonable.

Figures 5, 6, and 7 show fits to the three flare events using the new solution with $L=$ $2.7 \mathrm{AU}$, a value close to Burlaga's [1967] $L=$ $2.3 \pm 0.3 \mathrm{AU}$. It is apparent that the predictions of the new solution can be -made to agree very well with both the rise and decay

TABLE 1. Summary of the Flare Events Selected for Analysis

\begin{tabular}{|c|c|c|c|c|c|c|c|}
\hline & \multicolumn{3}{|c|}{ Optical Flare* } & \multirow{2}{*}{$\begin{array}{l}\text { UT of } \\
\text { X Ray } \\
\text { Flares* }\end{array}$} & \multirow{2}{*}{$\begin{array}{l}\text { UT of } \\
\text { Type IV } \\
\text { Radio* }\end{array}$} & \multirow{2}{*}{$\begin{array}{l}\text { Assumed UT } \\
\text { of Particle } \\
\text { Injection }\end{array}$} & \multirow{2}{*}{$\begin{array}{c}\text { Assumed † Avg. } \\
\text { Solas Wind } \\
\text { Velocity, } \\
\text { km/sec }\end{array}$} \\
\hline & Importance & $\begin{array}{c}\text { Solar } \\
\text { Coordinates }\end{array}$ & UT & & & & \\
\hline June 7, 1969 & $2 \mathbf{N}$ & $45^{\circ} \mathrm{E}, 15^{\circ} \mathrm{S}$ & $\begin{array}{l}0630-0730 \\
0806-0820 \\
0930-1100\end{array}$ & $?-1000$ & $0953-0959$ & 0800 & $400 \pm 50$ \\
\hline $\begin{array}{l}\text { Nov. } 2,1969 \\
\text { Jan. } 31,1970\end{array}$ & $\begin{array}{l}3 \mathbf{B} \\
\mathbf{2 B}\end{array}$ & $\begin{array}{l}90^{\circ} \mathrm{W}, 15^{\circ} \mathrm{N} \\
62^{\circ} \mathrm{W}, 22^{\circ} \mathrm{S}\end{array}$ & $\begin{array}{l}0939-1130 \\
1508-1535\end{array}$ & $\begin{array}{l}0945-1059 \\
1507-1544\end{array}$ & $1536-1614$ & $\begin{array}{l}1000 \\
1530\end{array}$ & $\begin{array}{l}325 \pm 70 \\
400 \pm 100\end{array}$ \\
\hline
\end{tabular}

* Taken from the Solar Geophysical Data (1969-1970).

$\dagger$ The errors are estimates of the variations of the actual solar wind velocity from that assumed for the calculation. 


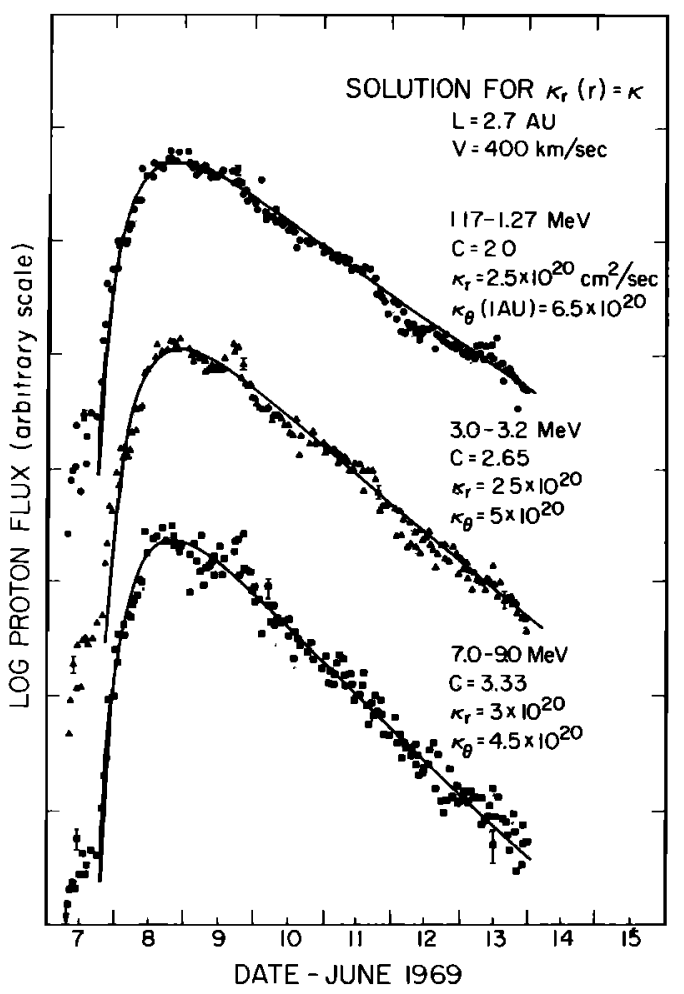

Fig. 5. Fits to the June 7, 1969, event using the present solution.

profiles of all three events at proton energies from $\sim 1 \mathrm{Mev}$ to $\sim 70 \mathrm{Mev}$.

Figures 8 summarizes the best-fit values of $\kappa_{r}$ for all three flare events for the assumed $L=2.7 \mathrm{AU}$. Since the value of $\kappa_{r}$ is largely determined by the rising part of the particle intensity-time profile, the derived $\kappa_{r}$ values are more likely characteristic of the magnetic field fluctuations inside $1 \mathrm{AU}$. The $\kappa$, values agree well with each other and are also of the same order of magnitude as the low-energy estimate for $\kappa_{\| 1}$ given by Jokipii and Coleman [1968] based on 1965 magnetic field measurements. The consistency between these three events is encouraging, especially since one of them (the June 7 event) was separated by $\sim 100^{\circ}$ in solar longitude from the foot of the near-earth field line, while the other two (Nov. 2 and Jan. 21) were west longitude events. The comparison with the magnetic field data should be regarded cautiously, since the magnetic field fluctuation in 1969-1970 may be different from that measured in 1965, and since the exact

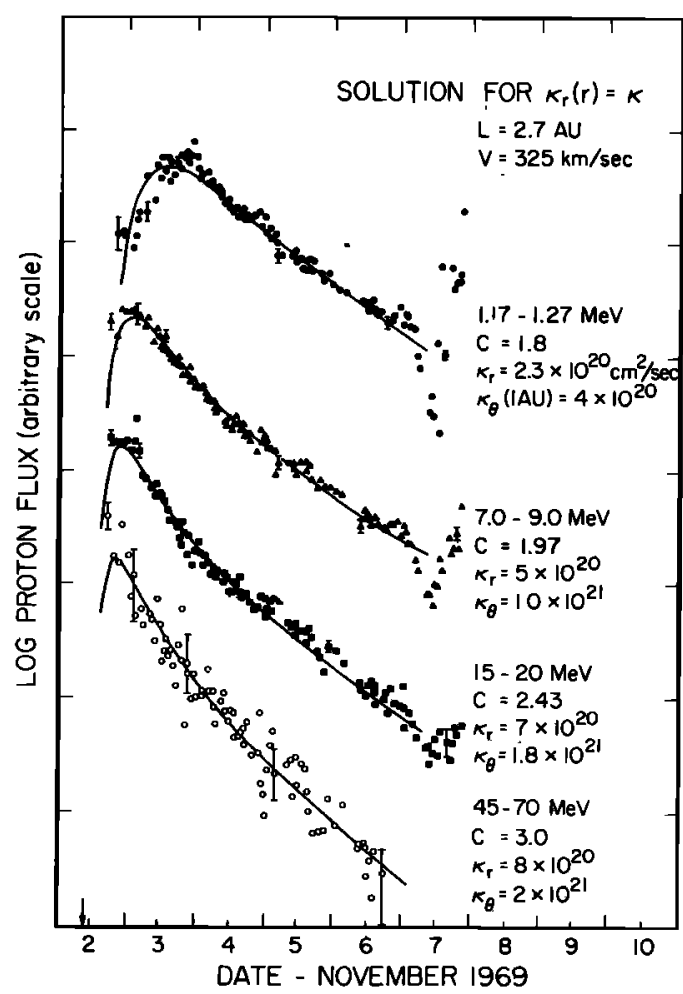

Fig. 6. Fits to the November 2, 1969, event using the present solution.

values of $\kappa_{r}$ derived from the fits to the particle data depend on the choice of other parameters, such as the boundary distance $L$ and the energy change effect.

The success of the solution at matching both the rise and decay times indicates that $\kappa_{r}(r)=$ constant is a better estimate of conditions inside $1 \mathrm{AU}$ than is $\kappa_{r}(r)=\kappa_{0} r$. The results are consistent with a $\kappa_{r}(r)$ constant out to a boundary at $2.7 \mathrm{AU}$, but other forms for the $r$ dependence of the diffusion coefficient beyond $1 \mathrm{AU}$ and corresponding changes in the boundary location cannot be excluded.

The interpretation of the azimuthal part of the solution is presently uncertain, partly because the experimental evidence is incomplete. However, there are a few comments that do seem appropriate at this time. For west limb events, the radial part of the solution alone adequately describes the abserved time profile at $1 \mathrm{AU}$. However, for the one east limb event observed some form of azimuthal propagation is necessary to explain the much slower rise time, 
which is not easily explained by corotation of a fixed azimuthal distribution with the $e$ folding angle of $\sim 30^{\circ}$ observed by McCracken et al. [1971]. One possibility suggested by Schatten and Fisk [1971] is that the azimuthal propagation takes place at $r \sim 1.5$ solar radii by means of rapid transport along current sheets in the corona. In that case, the fitted $\kappa_{\theta}$ values derived in this paper would not represent an interplanetary diffusion process.

Although the results of the fitting procedure presented here are consistent with the assumption that adiabatic deceleration is the only energy change process operative, other forms of energy change cannot be excluded.

\section{Particle ANisotropy}

It is also of interest to use the radial solution as the basis for an illustrative calculation of the vector particle anisotropy, which is

$$
\xi=\frac{3 \mathbf{S}}{U w}=\frac{3}{w}\left(C \mathbf{V}-\frac{1}{U} \kappa \cdot \nabla U\right)
$$

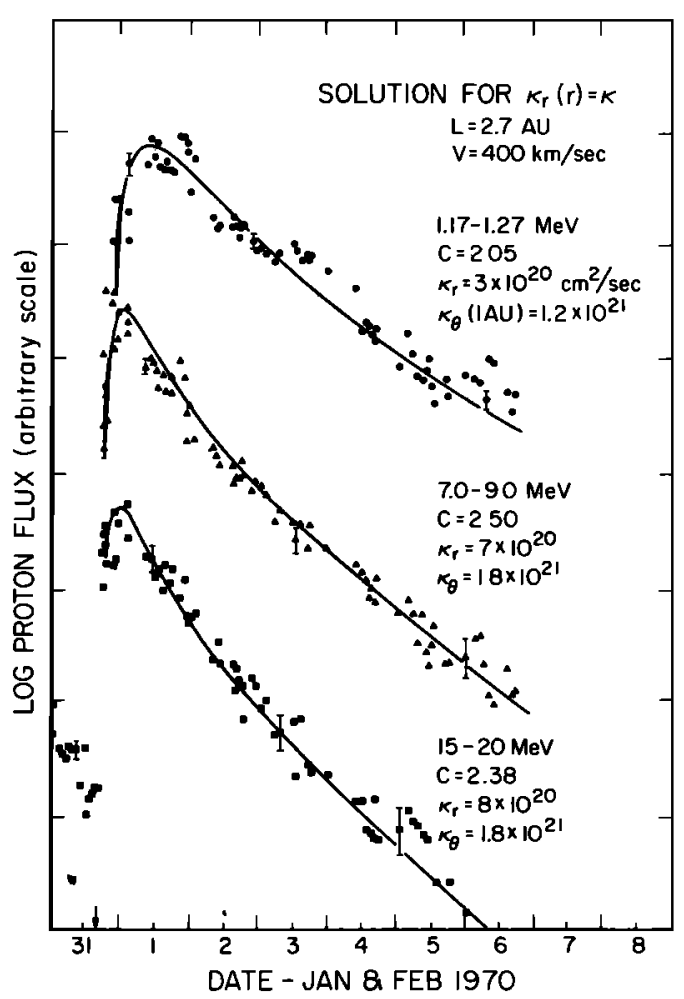

Fig. 7. Fits to the January 31, 1970, event using the present solution.

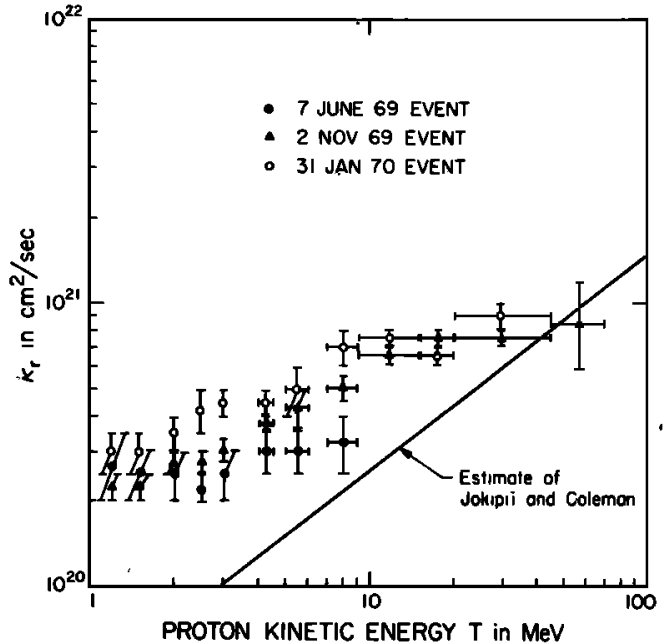

Fig. 8. The best-fit values of $\kappa_{r}$ are plotted versus proton energy $T$ for all three events for $L=2.7 \mathrm{AU}$. The $\kappa$ estimate of Jokipii and Coleman [1968] based on Mariner 4 field measurements is included for comparison.

where $w$ is the particle velocity. In order to investigate the vector properties of the anisotropy, we relax the assumption of a radial magnetic field and adopt the approach of $\mathrm{Ng}$ and Gleeson [1971b], who have taken the case $\kappa_{1}=0$, have assumed that azimuthal gradients are zero, and have taken $\kappa_{r}(r)=\kappa_{\|}(r) \cos ^{2} \psi$, where $\psi=\tan ^{-1}(\Omega r / V)$ is the spiral angle. This approach, which is an approximation to the

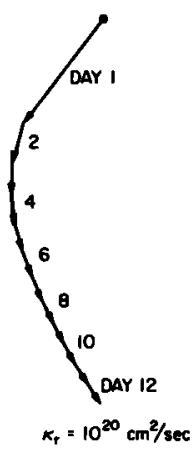

(a)

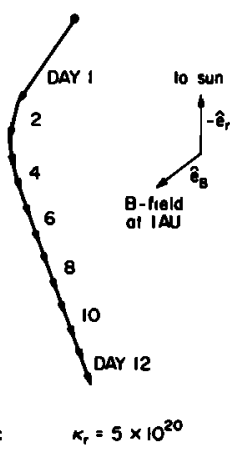

(b)

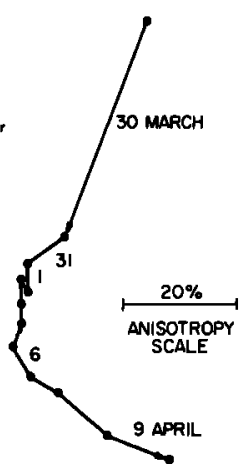

(c)
Fig. 9. A vector diagram of the time evolution of the anisotropy at 1-day intervals: ( $a$ and $b$ ) predictions of the radial solution for $L=2.3 \mathrm{AU}$, $V=400 \mathrm{~km} / \mathrm{sec}, C=2.0, r=1.0 \mathrm{AU}$, and $T_{\text {proton }}=10 \mathrm{Mev}$; (c) typical Pioneer 8 observation as reported by McCracken et al. [1971] for 7.5- to 21.5-Mev protons from March 30 to April 9, 1969. 
actual situation, uses the purely radial solution to characterize propagation along the spiral field. Thus, for constant $\kappa_{r}$ the $r$ dependence for $\kappa_{q}$ becomes $\kappa_{k}(r)=\kappa / \cos ^{2} \psi$, and the gradient along the field becomes $\nabla U=\cos \psi(\partial U / \partial r)$. The anisotropy can then be written as

$$
\begin{aligned}
\xi=\xi_{V} & +\xi_{\alpha} \\
& \equiv \frac{3}{w}\left(C V \hat{e}_{r}-\frac{\kappa}{\cos \psi} \frac{\partial R}{\partial r} \hat{e}_{B}\right)
\end{aligned}
$$

where $R(r, t)$ is the radial part of the solution, $\hat{e}_{r}$ is the radial unit vector, and $\hat{e}_{z}$ is the unit vector directed outward along the field $\left(\hat{e}_{\mathrm{r}}\right.$ and $\hat{e}_{B}$ meet at an angle $\psi \approx 48^{\circ}$ at $1 \mathrm{AU}$ ).

The vector anisotropy thus calculated is shown in Figure 9, which depicts the time evolution of $\xi$ in 1-day steps. Similar figures are given by $\mathrm{Ng}$ and Gleeson [1971b], and the nature of this variation has been discussed by Axford [1970]. A typical Pioneer 8 observation by $M c$ Cracken et al. [1971] is included for comparison. For 7- to 20-Mev protons at $\sim 1 \mathrm{AU}$, they observe a large 25 to $50 \%$ anisotropy early in the event directed from $\sim 45^{\circ} \mathrm{W}$, which decays to a value of 5 to $10 \%$ at late times directed approximately from $45^{\circ} \mathrm{E}$. Unfortunately, the relationship of the direction of these anisotropies to the field direction is not unambiguous, since no direct field measurements were reported. Therefore it was necessary to assume an average interplanetary field direction from $\sim 48^{\circ} \mathrm{W}$ in order to make a comparison with the calculations. The parameter values chosen for the calculation are typical for $\sim 10-\mathrm{Mev}$ protons, and there is qualitative agreement with the observations, e.g., $\xi$ decreases in magnitude and changes direction from the west to the east. The strength of the anisotropy predicted at late times is 3-8\% (depending on the value of $V, \kappa_{r}$, and $C$ chosen), a value which is in good agreement with the actual measurements. However, because of the approximations and assumptions made, this calculation is primarily illustrative.

A reasonable explanation for the anisotropy observed at $1 \mathrm{AU}$ can be made in terms of Figures 3 and 4. At late times, the typical equilibrium particle distribution has a peak beyond $1 \mathrm{AU}$. The diffusive component of the anisotropy at $1 \mathrm{AU}$ is thus directed inward along the field and produces an eastward anisotropy when combined with outward radial convective current. At early times the large density gradient (see Figure 1) produces a strong outward diffusive anisotropy along the field. This is exactly the description proposed by $\mathrm{McCracken}$ et al. [1971] and has been discussed in detail by $\mathrm{Ng}$ and Gleeson [1971b]. It should be emphasized, however, that variation in the field direction or the inclusion of perpendicular diffusion could alter the anisotropy should this be required when more detailed observations become available.

\section{Conchusions}

The solution presented here consists of resolving the differential equation for the radial part of the particle propagation, using $\kappa_{r}(r)=$ constant, and including the effects of convection and energy change known to be important at low energies. This new radial solution is capable of accurately reproducing both the rise and decay of west limb solar flare proton events observed at $1 \mathrm{AU}$ using reasonable values of the parameters. The principal limitations of the new radial solution are that it assumes a diffusion tensor that is independent of energy and diagonal in a reference frame aligned with the radial direction. Within these limitations, the comparison of the new solution with actual data leads to the following conclusions about solar flare particle propagation in the interplanetary medium:

(a) The diffusion tensor. The success of the present solution in fitting both the rise and decay of flare events indicates that $\kappa_{r}(r)=$ constant is a better approximation to actual conditions inside 1 AU than is $\kappa_{r}(r) \propto r$, which yields too slow a rise time [Forman, 1971a]. It is not necessary to invoke a scatter free region near the sun in order to reproduce the fast rise time observed for west limb flare events. The derived $\kappa_{r}$ values, which are most sensitive to conditions inside $1 \mathrm{AU}$, have a weak energy dependence and agree reasonably well with the estimates for $k_{\|}$based on Mariner 4 magnetic field measurements.

(b) Free escape boundary. The success of the present solution in fitting the decay phases of flare events indicates that the assumption of a free escape boundary at some 2 to $5 \mathrm{AU}$ is consistent with the observations at $1 \mathrm{AU}$. However, a sharp boundary may not exist, but there might instead be a finite region of rapidly increasing diffusion coefficient. 
(c) Anisotropy. Despite the limitations with respect to the actual spiral nature of the interplanetary field, the present solution can be used to understand many of the observed features of the vector particle anisotropy.

\section{Appendix A: Derivation of the New}

Solution to The Radial Equation

A solution $R(r, t)$ will be derived to the equation

$\frac{\partial^{2} R}{\partial r^{2}}+\left(\frac{2}{r}-\frac{V}{\kappa}\right) \frac{\partial R}{\partial r}-\frac{2 C V}{\kappa r} R=\frac{1}{\kappa} \frac{\partial R}{\partial t}$

using the assumptions and boundary conditions Jisted in the text.

It can easily be shown that if we write

$$
R(r, t)=y(r) \frac{1}{r} e^{\nabla r / 2 x} e^{-t / r}
$$

the function $y(r)$ is the solution to

$$
\frac{d^{2} y}{d r^{2}}+(\alpha-\beta / r) y=0
$$

where

$$
\begin{gathered}
\alpha=\frac{1}{\kappa \tau}-\frac{V^{2}}{4 \kappa^{2}}>0 \\
\beta=\frac{V}{\kappa}(2 C-1)>0
\end{gathered}
$$

A simple change of variable $\rho=(a)^{1 / 2} r$ reduces equation A3 to a form of the Coulomb wave equation,

$$
\frac{d^{2} y}{d \rho^{2}}+\left[1-\frac{\beta}{(\alpha)^{1 / 2}} \frac{1}{\rho}\right] y=0
$$

with solutions that are the regular and irregular Coulomb wave functions, $F_{0}\left(\beta / 2(\alpha)^{1 / 2},(\alpha)^{1 / 2} r\right)$ and $G_{0}\left(\beta / 2(\alpha)^{1 / 2},(\alpha)^{1 / 2} r\right)$. The general solution to equation $\mathrm{A} 1$ can then be written

$$
\begin{aligned}
& R(r, t)=\frac{\exp (V r / 2 \kappa)}{r} \\
& \quad \cdot \sum_{n}\left[A_{n} F_{0}\left(\beta / 2\left(\alpha_{n}\right)^{1 / 2},\left(\alpha_{n}\right)^{1 / 2} r\right)\right. \\
& \left.+B_{n} G_{0}\left(\beta / 2\left(\alpha_{n}\right)^{1 / 2},\left(\alpha_{n}\right)^{1 / 2} r\right)\right] \exp \left(-t / \tau_{n}\right)
\end{aligned}
$$

If we require that $R(r, t)$ remain finite as $r \rightarrow 0$, then $B_{n}=0$, and only the regular Cou- lomb functions are involved. The eigenvalues $\alpha_{n}$ are defined by the outer boundary condition $R(L, t)=0$. The eigenvalue equation for $\alpha_{n}$ is thus

$$
F_{0}\left(\beta / 2\left(\alpha_{n}\right)^{1 / 2},\left(\alpha_{n}\right)^{1 / 2} L\right)=0
$$

which must be solved by an iterative technique.

The coefficients $A_{n}$ are determined by the requirement of impulsive $\delta$ function injection at $r=r_{s}$ :

$$
R(r, t=0)=\frac{\delta\left(r-r_{\mathrm{s}}\right)}{r^{2}}
$$

We can thus write

$$
\begin{aligned}
R(r, 0) & =\frac{\delta\left(r-r_{s}\right)}{r^{2}} \\
& =\frac{\exp (V r / 2 \kappa)}{r} \sum_{n=1}^{\infty} A_{n} y_{n}(r)
\end{aligned}
$$

where

$$
y_{n}(r)=F_{0}\left(\beta / 2\left(\alpha_{n}\right)^{1 / 2},\left(\alpha_{n}\right)^{1 / 2} r\right)
$$

If we left-multiply by

$$
\int_{0}^{L} y_{m}(r) r \exp \left(-\frac{V}{2 \kappa} r\right) d r
$$

we have

$$
\begin{gathered}
\int_{0}^{L} y_{m}(r) \frac{\delta\left(r-r_{s}\right)}{r} \exp [-(V / 2 \kappa) r] d r \\
=\sum_{n=1}^{\infty} A_{n} \int_{0}^{L} y_{n}(r) y_{m}(r) d r
\end{gathered}
$$

In Appendix B the following orthogonality relationship for the regular Coulomb wave functions will be derived:

$$
\int_{0}^{L} y_{n}(x) y_{m}(x) d x=N_{m} \delta_{n m}
$$

where $\delta_{n m}$ is the Kronecker delta and $y_{n}(0)=$ $y_{n}(L)=0$. Using this relation, we can write

$$
\begin{aligned}
& y_{m}\left(r_{s}\right) \frac{\exp \left[-(V / 2 \kappa) r_{s}\right]}{r_{s}} \\
&=\sum_{n} A_{n} N_{m} \delta_{n m}=N_{m} A_{m}
\end{aligned}
$$

Thus 


$$
\begin{aligned}
A_{n}=\frac{\exp \left[-(V / 2 \kappa) r_{\mathrm{s}}\right]}{N_{n} r_{s}} \\
\cdot F_{0}\left[\beta / 2\left(\alpha_{n}\right)^{1 / 2},\left(\alpha_{n}\right)^{1 / 2} r_{s}\right]
\end{aligned}
$$

and the radial solution for the specific boundary conditions assumed becomes

$$
y_{m}(0)=y_{m}(L)=y_{n}(0)=y_{n}(L)=0
$$

the left side vanishes, and

$$
\int_{0}^{L} y_{n}(x) y_{m}(x) d x=N_{n} \delta_{m n}
$$

$$
\begin{aligned}
& R(r, t) \propto \frac{\exp \left[V\left(r-r_{s}\right) / 2 \kappa\right]}{r r_{e}} \\
& \qquad \sum_{n=1}^{\infty} \frac{F_{0}\left(\beta / 2\left(\alpha_{n}\right)^{1 / 2},\left(\alpha_{n}\right)^{1 / 2} r_{s}\right) F_{0}\left(\beta / 2\left(\alpha_{n}\right)^{1 / 2},\left(\alpha_{n}\right)^{1 / 2} r\right) \exp \left(-t / \tau_{n}\right)}{N_{n}}
\end{aligned}
$$

with

$$
\begin{gathered}
\beta=V(2 C-1) / \kappa \\
\tau_{n}=4 \kappa /\left(4 \kappa^{2} \alpha_{n}+V^{2}\right) \\
N_{n}=\int_{0}^{x}\left[F_{0}\left(\beta / 2\left(\alpha_{n}\right)^{1 / 2},\left(\alpha_{n}\right)^{1 / 2} x\right)\right]^{2} d x
\end{gathered}
$$

For $t \gg 1 / \kappa\left(\alpha_{2}-\alpha_{1}\right)$ the first term in equation A15 dominates and the function $R(r, t)$ decays exponentially with time. For typical values of the parameters $1 / \kappa\left(\alpha_{2}-\alpha_{1}\right) \sim 10$ hours.

Appendix B: The Orthogonaltit Relation for Regular Coulome Wave Functions

We wish to demonstrate that

$$
\int_{0}^{L} y_{n}(x) y_{m}(x) d x=0
$$

for $n \neq m$ given that $y_{n}(x)$ is the solution to

$$
\frac{d^{2} y_{n}}{d x^{2}}+\left(\alpha_{n}-\frac{\beta}{x}\right) y_{n}=0
$$

with boundary conditions $y_{n}(L)=y_{n}(0)=0$. We write down separate equations for two different eigenvalues $\alpha_{n}$ and $\alpha_{m}$, multiply by $y_{m}$ and $y_{n}$, respectively, and subtract:

$$
\begin{array}{r}
y_{m} y_{n}{ }^{\prime \prime}-y_{m}{ }^{\prime \prime} y_{n}=\left(\alpha_{m}-\alpha_{n}\right) y_{n} y_{m} \\
\frac{d}{d x}\left(y_{m} y_{n}{ }^{\prime}-y_{m}{ }^{\prime} y_{n}\right)=\left(\alpha_{m}-\alpha_{n}\right) y_{n} y_{m}
\end{array}
$$

If we left-multiply by $\int_{0}{ }^{L} d x$, we have

$$
\begin{aligned}
& {\left[y_{m} y_{n}^{\prime}-y_{m}{ }^{\prime} y_{n}\right]_{0}{ }^{L}} \\
& \quad=\left(\alpha_{m}-\alpha_{n}\right) \int_{0}^{L} y_{m} y_{n} d x
\end{aligned}
$$

But since
The problem remains to evaluate the normalization integral $N_{n}$. Using equation B4, one can write

$$
N_{n}=\operatorname{limit}_{\alpha_{m} \rightarrow \alpha_{n}} \frac{\left[y_{m} y_{n}{ }^{\prime}-y_{m}{ }^{\prime} y_{n}\right]_{0}{ }^{L}}{\alpha_{m}-\alpha_{n}}
$$

Applying l'Hôpital's rule we have

$$
\begin{aligned}
& N_{n}=\left\{\frac { \partial } { \partial \alpha _ { m } } \left[y\left(\alpha_{m}, x\right) y^{\prime}\left(\alpha_{n}, x\right)\right.\right. \\
&\left.\left.\quad-y^{\prime}\left(\alpha_{m}, x\right) y\left(\alpha_{n}, x\right)\right]_{\alpha_{m}=\alpha_{n}}\right\}_{0}^{L} \\
&= {\left[y_{\alpha}\left(\alpha_{n}, x\right) y^{\prime}\left(\alpha_{n}, x\right)\right.} \\
&\left.-y_{\alpha}{ }^{\prime}\left(\alpha_{n}, x\right) y\left(\alpha_{n}, x\right)\right]_{0}^{L}
\end{aligned}
$$

where

$$
y\left(\alpha_{n}, x\right)=y_{n}(x)
$$

$$
y_{\alpha}\left(\alpha_{n}, x\right)=\left.\frac{\partial}{\partial \alpha} y(\alpha, x)\right|_{\alpha=\alpha_{n}}
$$

However,

$$
\begin{gathered}
y\left(\alpha_{n}, 0\right)=y\left(\alpha_{n}, L\right)=y_{\alpha}\left(\alpha_{n}, 0\right)=0 \\
N_{n}=y_{\alpha}\left(\alpha_{n}, L\right) y^{\prime}\left(\alpha_{n}, L\right)
\end{gathered}
$$

In terms of the Coulomb wave functions, this becomes

$N_{n}=\left[\frac{x}{2}\left(F_{0}^{\prime}\right)^{2}-\frac{\beta}{4 \alpha_{n}} F_{0}^{\prime} \frac{\partial F_{0}}{\partial \eta}\right]_{x=L}$

where

$$
F_{0}(\eta, \rho)=F_{0}\left[\beta / 2\left(\alpha_{n}\right)^{1 / 2},\left(\alpha_{n}\right)^{1 / 2} x\right]
$$

and

$$
F_{0}^{\prime}=\partial F_{0} / \partial \rho
$$


Equation B9 isn't a simple evaluation of $N_{n}$, but it greatly facilitates the calculation because time-consuming numerical integration can be avoided. A useful discussion of the methods for calculating the Coulomb wave functions has been given by Fröberg [1955].

Acknowledgments. We are grateful to R. E. Vogt who has been intimately involved with the Ogo 6 experiment. We also appreciate useful discussions with J. R. Jokipii and S. S. Murray. This work was supported in part by the National Aeronautics and Space Administration under contract NAS5-9312 and Grants NGR-05-002-160 and NGL-05-002-007. One of us (Lupton) has received valuable support from a NDEA fellowship and a NASA traineeship, and the other (Stone) was an Alfred $P$. Sloan research fellow during this work.

The Editor wishes to thank R. C. Englade and L. J. Gleeson for their assistance in evaluating this paper.

\section{REFERENCES}

Abramowitz, M., and I. A. Stegun, Handbook of Mathematical Functions, p. 538, Dover, New York, 1964.

Althouse, W. E., E. C. Stone, R. E. Vogt, and T. H. Harrington, A solar and galactic cosmic ray satellite experiment, IEEE Trans. Nucl. Sci., 15, 229, 1967.

Axford, W. I., A review of theoretical work on the effects of solar wind transport on energetic solar particles, presented at Seminar on Cosmic Ray Generation on the Sun, USSR Acad. Sci., Leningrad, December 1970.

Burlaga, L. F., Anisotropic diffusion of solar cosmic rays, J. Geophys. Res., 72, 4449, 1967.

Englade, R. C., A computational model for solar flare particle propagation, J. Geophys. Res., 76, 768, 1971a.

Englade, R. C., Effects of the solar boundary condition on flare-particle propagation, J. Geophys. Res., 7.6, 6190, $1971 b$.

Fisk, L. A., and W. I. Axford, Effect of energy changes on solar cosmic rays, J. Geophys. Res., 73, 4396, 1968.
Forman, M. A., The equilibrium anisotropy in the flux of $10 \mathrm{Mev}$ solar flare particles and their convection in the solar wind, J. Geophys. Res., 75, 3147, 1970.

Forman, M. A., Convection-dominated transport of solar cosmic rays, J. Geophys. Res., 76, 759, $1971 a$.

Forman, M. A., Convection-dominated transport of solar cosmic rays, paper presented at the 12th International Conference on Cosmic Rays, Hobart, Tasmania, August $1971 b$.

Fröberg, C. E., Numerical treatment of Coulomb wave functions, Rev. Mod. Phys., $27,399,1955$.

Gleeson, L. J., and W. I. Axford, Cosmic rays in the interplanetary medium, Astrophys. J., 149, L115, 1967.

Jokipii, J. R., and P. J. Coleman, Jr., Cosmic ray diffusion tensor and its variation observed with Mariner 4, J. Geophys. Res., 73, 5495, 1968.

Jokipii, J. R., and E. N. Parker, Energy changes of cosmic rays in the solar system, Planet. Space Sci., $15,1375,1967$.

Lupton, J. E., and E. C. Stone, Transport of solar flare protons: Comparison of a new analytic model with spacecraft measurements, paper presented at the 12th International Conference on Cosmic Rays, Hobart, Tasmania, August 1971.

McCracken, K. G., U. R. Rao, R. P. Bukata, and E. P. Keath, The decay phase of solar flare events, Solar Phys., 18, 100, 1971.

$\mathrm{Ng}$, C. K., and L. J. Gleeson, On the propagation of solar cosmic rays, paper presented at the 12th International Conference on Cosmic Rays, Hobart, Tasmania, August 1971a.

$\mathrm{Ng}, \mathrm{C}$. K., and L. J. Gleeson, The propagation of solar cosmic ray bursts, Solar Phys., 20, 166, $1971 b$.

Parker, E. N., The passage of energetic charged particles through interplanetary space, Planet. Space Sci., 13, 9, 1965.

Schatten, K. H., and L. A. Fisk, Transport of cosmic rays in the solar corona (abstract), Eos Trans. $A G U, 62,889,1971$.

(Received March 14, 1972; accepted October 30, 1972.) 